\title{
Comparación del Neurocráneo entre el Gato Doméstico (Felis catus Linneas 1758) y el Montés (Felis silvestris Schreber, 1777) Mediante Técnicas de Morfometría Geométrica
}

\author{
Comparison of Neurocranium Between Domestic Cat (Felis catus Linneas 1758) and Wild \\ Cat (Felis silvestris Schreber, 1777) by Means of Geometric Morphometric Techniques
}

Pere M. Parés-Casanova

\begin{abstract}
PARÉS-CASANOVA, P. M. Comparación del neurocráneo entre el gato doméstico (Felis catus Linneas 1758) y el montés (Felis silvestris Schreber, 1777) mediante técnicas de morfometría geométrica. Int. J. Morphol., 39(3):823-828, 2021.

RESUMEN: El objetivo de este estudio fue comparar objetivamente las diferencias en la forma total (tamaño y forma pura) del neurocráneo entre un grupo de cráneos de gatos domésticos (Felis catus, $\mathrm{n}=81$ ), y un grupo de gato montés (Felis silvestris, $\mathrm{n}=12$ ) mediante técnicas de morfometría geométrica. Para ello se obtuvieron fotografías digitales del lado izquierdo de los cráneos de un modo estandarizado, y en las que se ubicaron 8 landmarks y 9 semilandmarks. Después de una superposición generalizada de Procrustes se procedió a un análisis de componentes principales y a otro de canónico a fin de comparar y detectar las posibles diferencias. Los tamaños neurocraneales no mostraron diferencias entre ambos grupos, pero sí que aparecieron diferencias significativas en la forma. Los cambios de forma afectarían principalmente la sutura escamoso-parietal-alisfenoidal y, en menor medida, puntos caudales del cráneo, lo que sugeriría cambios a nivel de músculos masticatorios. A conocimiento del autor, constituye esta la primera investigación que caracteriza y compara el cráneo de los gatos mediante métodos de morfometría geométrica, recomendándose un mayor uso de esta técnica dada la escasez de publicaciones al respecto en mamíferos domésticos.
\end{abstract}

PALABRAS CLAVE: Cráneo; Domesticación; Felidae.

\section{INTRODUCCIÓN}

En la actualidad se conocen 23 subespecies de Felis silvestris (Integrated Taxonomic Information System Online Database, 2020) y se los considera los antecesores de los gatos domésticos (Ottoni et al., 2017). El gato montés europeo pertenece al grupo Felis silvestris Schreber, 1775 presenta una distribución muy amplia, desde Europa Occidental (España y Portugal) hasta el Cáucaso (García-García, 2004). Se considera comúnmente queel gato doméstico (Felis catus Linneas 1758) desciende del gato montés norteafricano o Felis silvestris líbica (García-García; Hu et al., 2014; Serpell, 2014; Ottoni et al.), una subespecie que se encuentra en el norte de África y el Cercano Oriente (Vigne et al., 2016).

Se postula que esta domesticación ocurrió durante el Neolítico (Ottoni et al.), en manos de los primeros agricultores en el Cercano Oriente, aunque la domesticación propiamente dicha, con la aceptación de gatos en las casas como animal de compañía, se dio en Egipto cuando se valoró su papel en el control de roedores y serpientes (Serpell), hace unos 4.000 años (Hu et al.). El gato empezó entonces a formar parte de la vida de los humanos y a lo largo del siglo VIII se extendió por todo Occidente (Ottoni et al.). La selección artificial se basó factores estéticos (MenottiRaymond et al., 2008), y se produjeron finalmente diferenciaciones en razas (Menotti-Raymond et al.) a partir del medioevo (Ottoni et al.).

El gato montés tiene la apariencia de un gato doméstico atigrado de tamaño grande, aunque con menos rayas y menos conspicuas que el doméstico. Es un animal robusto, de rostro corto y cabeza masiva y ancha, compacta, con apariencia robusta y fuerte (García-García). En el gato doméstico, una diferencia desde el punto de vista de la morfología externa es la reducción del tamaño de la cabeza (Stefen \& Heidecke, 2011). 
El objetivo de este trabajo es realizar un estudio morfométrico del cráneo en gato doméstico (Felis catus) con el montés (Felis silvestris) aplicando métodos de morfometría geométrica (MG). Aunque ambas especies pueden diferenciarse por un índice de capacidad craneana (García-García), la MG permite información sobre forma pura, algo que un índice no refleja.Por tanto, la investigación no pretende tener un carácter diagnóstico sino de contribución a la descripción puramente morfológica entre ambos Felis y, a la vez, intentar inferir los cambios derivados del proceso de domesticación.

\section{MATERIAL Y MÉTODO}

Se estudió un total de 93 cráneos de Felis, unos pertenecientes a gatos montés (Felis silvestris, $\mathrm{n}=12$ ), y el resto $(\mathrm{n}=81)$ a gatos domésticos(Felis catus $)$ de raza indefinida. Los ejemplares monteses están depositados en la colección del Museu de Zoologia de Barcelona (Cataluña), y los domésticos, una parte procede del Museo de Ciència de la Universidade de Coimbra (Portugal) y el resto, de la colección fotográfica propia de la Universitat de Lleida (Cataluña). En todos los casos, los cráneos estaban desarticulados de la correspondiente mandíbula.

Todos los especímenes estudiados pertenecen a animales adultos - con una fusión completa de la sincondrosis esfeno-occipital. Ninguno de ellos presentaba patología aparente o deformaciones craneales y en el caso de los domésticos, ningún espécimen presentaba formas cefálicas extremas (braqui o dolicocefalismo), carácter éste que también se refleja en la conformación neurocraneal (Künzel et al., 2003). Puesto que de la mayoría de especímenes se desconocía la edad y el sexo, estos parámetros no se tuvieron en cuenta para el presente trabajo, aunque ello no debería tampoco interferir en los resultados por la ausencia de dimorfismo sexual craneal en esta especie (Pitakarnnop et al., 2017).

Se obtuvieron fotografías digitales en el plano lateral izquierdo de cada espécimen con una cámara digital Nikon (D5100) equipada con un objetivo AF-S DX Micro Nikkor 40mm f/1·2.8G. Los cráneos fueron fijados con plastilina para facilitar que quedaran paralelos al plano focal de la cámara. Todas las fotografías incluyeron una escala de referencia. Un total de 8 landmarks fueron seleccionados para caracterizar cada espécimen, y concretamente, en el neurocráneo. Al considerarse que la curvatura dorsal parietal podía ser un punto anatómico crucial en la descripción de los ejemplares, se consideraron igualmente 9 semilandmarks a lo largo de la curvatura dorsal parietal. A diferencia de los landmarks, los semilandmarks (también llamados puntos de referencia de deslizamiento) son puntos discretos que se obtienen inicialmente como coordenadas en una curva inicial, para después transformarse en puntos discretos equidistantes (Webster \& Sheets, 2010). Los landmarks y semilandmarks se obtuvieron en cada imagen con la aplicación tpsDig2 v. 2.16 (Rohlf, 2015), introduciendo el factor de escala en el fichero digitalizado. Los landmarks y semilandmarks seleccionados se encuentran en la Figura 1. Con el programa tps Utility v. 1.70 (Rohlf) se optimizaron los semilandmarks mediante el proceso sliding landmarks. Se procesaron posteriormente mediante el método Procrustes obteniendo un consenso, la media Procrustes, que minimiza la distancia entre configuraciones de landmarks, y se ha obtenido una matriz de varianza-covarianza.

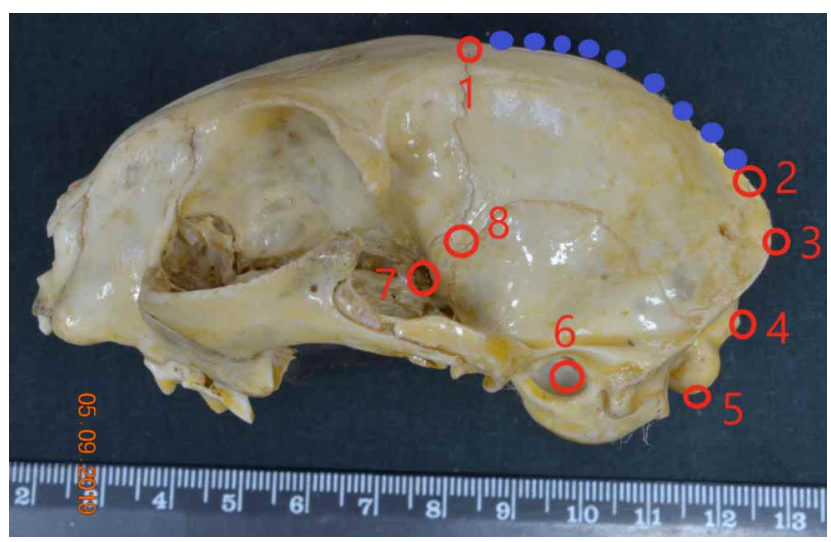

Fig. 1. Puntos anatómicos (landmarks y semilandmarks) utilizados en este estudio de cráneos de Felis, en el plano lateral. Un total de 9 semilandmarks (que aparecen sin numerar como puntos rellenos) definieron la curvatura dorsal parietal.

1 Punto más dorsal de la sutural coronal

2 Punto más dorsal de la sutura parieto-interparietal

3 Punto más caudal del borde lamboidal

4 Punto más caudo-dorsal del cóndilo occipital

5 Punto más ventral del cóndilo occipital

6 Punto medio del poro acústico externo

7 Foramen óptico

8 Punto más craneal de la sutura escamoso-parietal-alisfenoidal

Se ha definido la "forma pura" como la información geométrica que permanece en un objeto cuando se han extraído o normalizado el tamaño, la traslación y la rotación (Zelditch et al., 2004). Así pues, con el fin de eliminar las distorsiones de esos componentes no relacionados con la forma pura, las configuraciones de los landmarks se sobreimpusieron mediante un análisis generalizado de Procrustes, método basado en la minimización generalizada de cuadrados mínimos de las distancias entre puntos (Bookstein, 1991). Mediante esta 
sobreimposición, cada configuración se traslada a una posición común de centroide, se escala a un tamaño de centroide uno, y se rota a fin de minimizar las distancias entre los landmarks equivalentes. Todo ello permitió separar las variables "forma pura" y "tamaño".

Para evaluar los efectos del error intraobservacional, se realizó una segunda obtención de las coordenadas. Para evaluar si la variación entre los dos espacios (euclideano y tangente) era mínimo, también se computaron las correlaciones entre las distancias tangentes y las distancias Procrustes mediante la aplicación tpsSmall v. 1.33 (Rohlf). El resultado de esta correlación $(0,999998)$ confirmó que ambos espacios eran casi idénticos.

La comparación de tamaños se analizó mediante un test U de Mann-Whitney comparando el tamaño de centroide para los dos grupos. El tamaño del centroide se define como la raíz cuadrada del sumatorio de las distancias al cuadrado entre cada landmark y el centroide (Bookstein). Se realizó igualmente un análisis de regresión multivariado, en el que la matriz de las variables de forma fue usada como variable dependiente y el logaritmo del tamaño del centroide como variable independiente (Gould, 1966). Se generó así una matriz de covarianza de los residuales a partir de la cual se realizó un Análisis de Componentes Principales (ACP). En el $\mathrm{ACP}$, el primer componente principal puede interpretarse como un índice del tamaño y el Segundo, como un índice de forma. Se realizó seguidamente un Análisis Canónico Variante (ACV) para mejorar la distinción entre los grupos evaluados, usando la distancia de Mahalanobis y una ronda de 1,000 permutaciones.Y, finalmente, se compararon los patrones de covariación entre los dos grupos, para el módulo "caudo-ventral" (landmarks 3 a 8) y para el módulo "dorsal" (landmarks 1 y 2 , y todos los semilandmarks), los cuales fueron cuantificados usando el coeficiente de RV (Klingenberg, 2014). Y, finalmente, mediante un thin plate spline los cambios de forma promedio entre los dos grupos, con lo que pudo interpretarse visualmente estos cambios (donde estos cambios de forma se aprecian como fuerzas que actúan sobre una malla que se deforma según el sentido: expansión o contracción). Este procedimiento permitió apreciar los cambios de forma como fuerzas que actúan sobre una malla que se deforma según el sentido (positivo o negativo).

Los análisis se realizaron con los paquetes estadísticos MorphoJ v. 1.6.0c (Klingenberg, 2011) y PAST v. 2.17 c (Hammer et al., 2001). El nivel de significación estadístico se estableció en el $95 \%$.

\section{RESULTADOS}

Error observacional. El porcentaje de error entre réplicas para el tamaño resultó ser del 0,6\%, muy por debajo del $5 \%$ que se establece para este tipo de estudios (von Cramon-Taubadel et al., 2007), y sin diferencias estadísticamente significativas entre ambas tomas ( $\mathrm{F} 2=0,569 ; \mathrm{p}=0,556)$.

Diferencias entre grupos. No se detectaron diferencias en los tamaños del centroide entre los dos grupos $(\mathrm{U}=1612$; $\mathrm{p}=0,178$ ) aunque el grupo doméstico presentó mucha más variación que el montés (Fig. 2). La regresión resultó ser estadísticamente significativa $(\mathrm{p}<0,001)$, por lo que para los siguientes análisis se utilizaron los "scores" de la regresión. A partir de los valores para los tres primeros Componentes Principales $(\mathrm{CP})(\mathrm{CP} 1+\mathrm{CP} 2+\mathrm{CP} 3=25,11$ $\%+22,20 \%+12,51 \%=59,83 \%)$. Los valores de descarga fueron tanto positivos como negativos (Tabla I), lo que refuerza que las diferencias entre los dos grupos no recaen en el tamaño si no en el tamaño. Los caracteres más discriminantes se localizarona nivel de la sutura escamosoparietal-alisfenoidal (x8, y8), con desplazamientos en el plano horizontal y vertical y, en menor contribución, parte más caudal de la curvatura dorsal parietal (x13, x14 e x17), margenlamboidal (x3) y cóndilo occipital (x5), con desplazamientos horizontales. El ACV reveló diferencias significativas entre ambos grupos ( $\mathrm{p}<0,0001)$ (Fig. 3), expresadas como una expansión caudal y una contracción

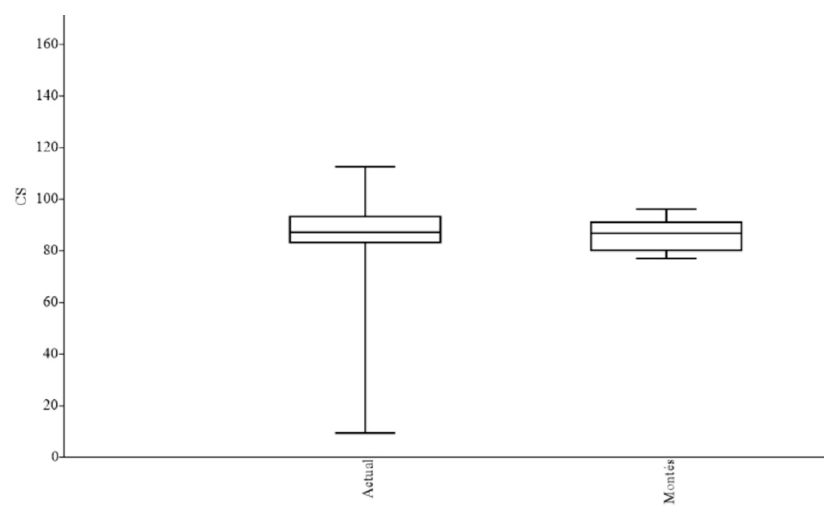

Fig. 2. Diagrama de caja para el tamaño (expresado como tamaño de centroide) para 12 cráneos de Felis silvestris y 81 de Felis catus). La mediana de viene representada por el valor central dentro de cada caja, que representa el $50 \%$ central de las puntuaciones de una variable, sin el $25 \%$ más pequeño y el $25 \%$ más alto representados por los "bigotes" superior e inferior. No se detectaron diferencias en los tamaños del centroide entre los tres grupos ( $U=1612 ; p=0,178$ ), aunque el grupo doméstico presentó mucha más variación que el montés. 
basicraneal en el gato doméstico en relación al montés (Fig. 4). El RV para el montés fue de 0,759 y para el doméstico, de 0,377 , de lo que se deduce un crecimiento modular más isométrico en el primer grupo.

\section{DISCUSION}

La craneometría puede usarse para determinar elementos biológicos en los mamíferos domésticos (Sañudo, 2009). Aunque en el estudio de la domesticación del perro se ha usado mucho la morfometría geométrica en cráneo (Drake \& Klingenberg, 2010; Drake et al., 2015, 2017), de la domesticación del gato se tiene poca información (Finka et al., 2019).

Aunque la subespecie de gato montés no corresponde al agriotipo del gato doméstico, podemos suponer que las variaciones a nivel de cráneo en Felis silvestris son escasas, por lo que podemos inferir algunas conclusiones sobre el proceso de domesticación del gato. Los resultados del presente trabajo muestran unas diferencias significativas en la forma -pero no en el tamaño- del neurocráneo entre gatos domésticos y monteses. Las diferencias son regionales, estando focalizadas enalgunos puntos del neurocráneo, sobre todoa nivel de la sutura escamoso-narietal-alisfenoidal. $\mathrm{v}$ en menor medida. en

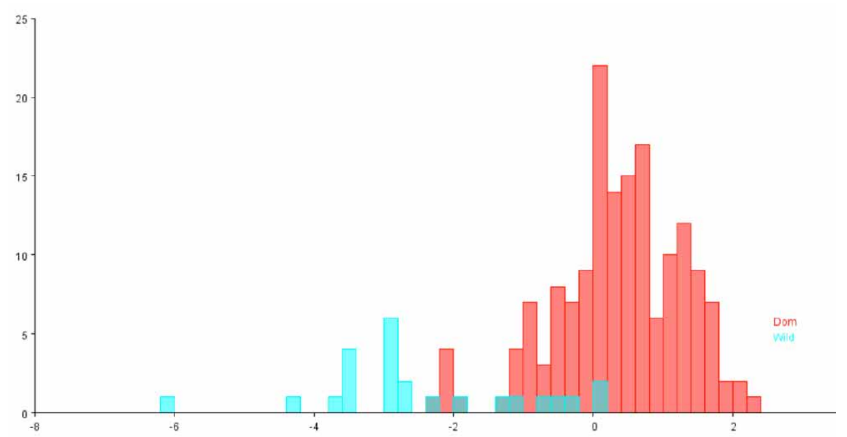

Fig. 3. Representación gráfica del Análisis Canónico Variante para los 91 cráneos estudiados de Felis adultos (12 monteses, Felis silvestris"Wild"; 81 domésticos, Felis catus "Dom"). Aunque existe una pequeña superposición entre los morfoespacios de los dos grupos, se presentaron diferencias estadísticamente significativas entre ambos $(\mathrm{p}<0,0001)$.

los puntos más caudales del cráneo, lo que corresponde a áreas de inserción de músculos masticatorios, sobre todo el músculo temporal (Barone, 2000; Sebastiani \& Fishbeck, 2005).

El proceso de domesticación del gato, pues, a nivel del neurocráneo, parecería haber conllevado además un alargamiento a nivel basal y un acortamiento a nivel dorsal. Se demuestra pues una plasticidad regional a nivel neurocraneal con cambios relacionados con requerimientos funcionales de la masticación. Pero a nuestro

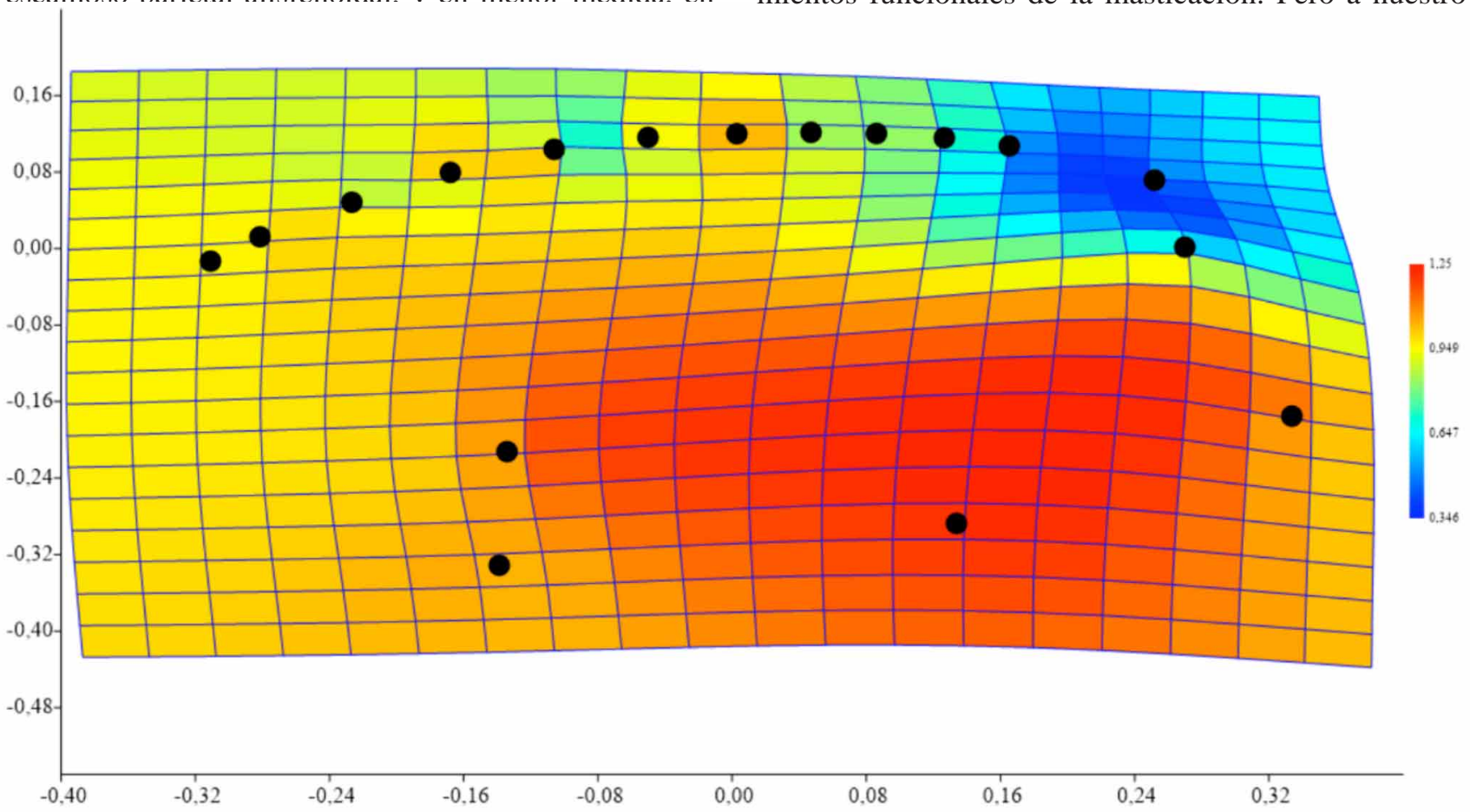

Fig. 4. Thin plate spline. Deformaciones parciales del paso del cráneo de gato montés al doméstico, en las que la malla se deforma según el sentido (expansión o contracción, en tonos rojizos o azules, respectivamente), mostrando una expansión dorsal caudal y una contracción basicraneal en el gato doméstico en relación al montés. 
Tabla I. Matriz de estructura de los tres primeros Componentes Principales (CP) para las coordenadas de los 8 landmarks y 9 semilandmarks utilizados, que explicaron un $59,83 \%$ de la variación total observada $(\mathrm{CP} 1+\mathrm{CP} 2+\mathrm{CP} 3=25,11 \%+22,20 \%+12,51$ $\%)$. Se resaltan las variables con el máximo de valor de descarga $(>[0,3]$

\begin{tabular}{|c|c|c|c|}
\hline Variables & CP1 & $\mathrm{CP} 2$ & CP3 \\
\hline $\mathrm{x} 1$ & 0,10483 & 0,18299 & $-0,01498$ \\
\hline y 1 & $-0,27509$ & 0,13833 & $-0,04427$ \\
\hline $\mathrm{x} 2$ & 0,15789 & 0,23537 & $-0,07476$ \\
\hline y2 & $-0,12836$ & $-0,21122$ & $-0,18501$ \\
\hline $\mathbf{x} 3$ & $-0,39328$ & 0,11737 & 0,23933 \\
\hline y3 & 0,06695 & $-0,11000$ & 0,02753 \\
\hline $\mathrm{x} 4$ & 0,18674 & 0,08955 & 0,03703 \\
\hline y4 & 0,17267 & 0,12011 & 0,05992 \\
\hline $\mathbf{x} 5$ & 0,08335 & 0,16465 & $-0,35128$ \\
\hline y5 & $-0,10861$ & $-0,12039$ & 0,01868 \\
\hline$x 6$ & 0,07767 & 0,27726 & $-0,03852$ \\
\hline y6 & 0,01324 & $-0,17559$ & $-0,09106$ \\
\hline$x 7$ & $-0,01402$ & 0,15199 & 0,08982 \\
\hline y7 & $-0,08221$ & 0,13866 & 0,27613 \\
\hline $\mathbf{x} 8$ & 0,15309 & 0,13025 & 0,44236 \\
\hline y8 & $-0,34472$ & 0,42975 & $-0,53853$ \\
\hline x9 & 0,18863 & 0,06146 & 0,00267 \\
\hline y9 & 0,17570 & 0,09448 & 0,05133 \\
\hline$\times 10$ & 0,18583 & $-0,04913$ & $-0,10710$ \\
\hline y 10 & 0,16763 & 0,00729 & 0,00839 \\
\hline $\mathrm{x} 11$ & 0,18639 & $-0,14449$ & $-0,17033$ \\
\hline y 11 & 0,12787 & $-0,04223$ & 0,01353 \\
\hline $\mathrm{x} 12$ & 0,08748 & $-0,24967$ & $-0,16037$ \\
\hline y 12 & 0,06133 & $-0,05429$ & 0,02828 \\
\hline $\mathbf{x 1 3}$ & $-0,01781$ & $-0,33446$ & $-0,15899$ \\
\hline y 13 & 0,02726 & $-0,04743$ & 0,05242 \\
\hline $\mathbf{x 1 4}$ & $-0,12249$ & $-0,34174$ & $-0,06346$ \\
\hline y 14 & 0,01793 & 0,00050 & 0,06748 \\
\hline $\mathrm{x} 15$ & $-0,20373$ & $-0,22788$ & 0,00587 \\
\hline y 15 & 0,02729 & $-0,03451$ & 0,08026 \\
\hline x 16 & $-0,2976$ & $-0,09035$ & 0,08871 \\
\hline y 16 & 0,03647 & $-0,04656$ & 0,10445 \\
\hline $\mathbf{x 1 7}$ & $-0,36302$ & 0,026816 & 0,23396 \\
\hline у 17 & 0,04460 & $-0,08694$ & 0,07040 \\
\hline
\end{tabular}

parece, estos cambios morfológicos se deberían a cambios en la función masticatoria. Se postula que la formación de las razas en el gato se ha basado sobre todo en factores visibles, estéticos (Menotti-Raymond et al.), pero a nuestro parecer, con los resultados obtenidos, parecería que se habría dado un cierto "sesgo", muy probablemente de manera no intencionada puesto que no ha habido selección por función.Así pues, la domesticación del gato, al haber implicado un importante cambio de entorno (el paso de un entorno salvaje a uno de antropizado), se habría reflejado como cambios morfológicos en las zonas de origen/inserción de los paquetes musculares relacio- nados con la domesticación, puesto que hemos de tener en cuenta que en el cráneo y, en particular, en sus complejos óseos, se muestra una clara relación entre estructura y función (Stacharski et al., 2015), por. O sea, cambios meramente derivados del nuevo entorno humano en el que forjó el gato doméstico, y no únicamente cambios tróficos; se han descrito cambios por entorno en el cráneo de zorro rojo (Vulpes vulpes) de zonas urbanas en relación al de zonas rurales (Parsons et al., 2020), por lo que nuestra consideración no es nada descabellada. Recordemos por otro lado que en la muestra analizada no habían cráneos de morfología extrema (como los que aparecen en algunas razas breviprosopias, como el Persa, Birmano o Himalaya), por lo que, de nuevo, los cambios morfológicos observados no se consideran haberse debido a un motivo de selección artificial directa.

Por otro lado, de nuestros datos aparece que el gato montés exhibe un crecimiento más isométrico que el doméstico, lo que igualmente está en consonancia con lo expuesto por otros autores (Stefen \& Heidecke, 2012; Sánchez-Villagra et al., 2017).

Investigaciones futuras deberían considerarlos cambios morfométricos puedan asociarse al esplacnocráneo, y que seguramente permitirían una descripción complementaria y seguramente mucho más informativa en el proceso de domesticación del gato (Künzel et al.).

\section{AGRADECIMIENTOS}

Los autores agradecemos a Javier Quesada y Joana Pedroso de Lima Cabral de Oliveira, por habernos permitido el acceso a la colección mastozoológica del Museu de Zoologia de Barcelona (Cataluña) y del Museo de Ciència de la Universidade de Coimbra (Portugal), respectivamente. Agradecemos igualmente las observaciones aportadas por los revisores de la Revista.

PARÉS-CASANOVA, P. M. Comparison of neurocranium between domestic cat (Felis catus Linneas 1758) and wild cat (Felis silvestris Schreber, 1777) by means of geometric morphometric techniques. Int. J. Morphol., 39(3):823-828, 2021.

SUMMARY: The objective of this study was to objectively compare the differences in the total shape (size and pure shape) of the neurocranium between a group of domestic cat skulls (Felis catus, n=81), and wildcats (Felis 
silvestris, $\mathrm{n}=12$ ) by means of geometric morphometric techniques. To achieve it, we obtained digital photographs of the left side of the skulls in a standardized form, in which 8 landmarks and 9 semilandmarks were located. After a Generalized Procrustes Superimposition, a Principal Component Analysis and a Canonical Analysis one were performed to compare and detect possible differences. Neurocranial sizes showed no differences between the two groups, however, significant differences in shape did appear. Shape changes would mainly affect the squamosal-coronal suture and, to a lesser extent, caudal skull points, therefore suggesting changes in masticatory muscles. As far as this author's knowledge, this is the first research that characterizes and compares the skull of cats using geometric morphology methods, recommending greater use of this technique given the scarcity of publications on this subject in domestic mammals.

\section{KEY WORDS: Domestication; Felidae; Skull.}

\section{REFERENCIAS BIBLIOGRÁFICAS}

Barone, R. Anatomie Comparée des Mammifères Domestiques. Tome 2. Arthrologie et Myologie. Paris, Vigot, 2000.

Bookstein, F. L. Morphometric Tools for Landmark Data: Geometry and Biology. Cambridge (UK), Cambridge University Press, 1991.

Drake, A. G. \& Klingenberg, C. P. Large-scale diversification of skull shape in domestic dogs: disparity and modularity. Am. Nat., 175(3):289-301, 2010.

Drake, A. G.; Coquerelle, M. \& Colombeau, G. 3D morphometric analysis of fossil canid skulls contradicts the suggested domestication of dogs during the late Paleolithic. Sci. Rep., 5:8299, 2015.

Drake, A. G.; Coquerelle, M.; Kosintsev, P. A.; Bachura, O. P.; Sablin, M.; Gusev, A. V.; Fleming, L. S. \& Losey, R. J. Three-dimensional geometric morphometric analysis of fossil canid mandibles and skulls. Sci. Rep., 7(1):9508, 2017.

Finka, L. R.; Luna, S. P.; Brondani, J. T.; Tzimiropoulos, Y.; McDonagh, J.; Farnworth, M. J.; Ruta, M. \& Mills, D. S. Geometric morphometrics for the study of facial expressions in non-human animals, using the domestic cat as an exemplar. Sci. Rep., 9(1):9883, 2019.

García-García, F. El gato montés Felis silvestris Schreber, 1775. Galemys, 16(1):1-14, 2004.

Gould, S. J. Allometry and size in ontogeny and phylogeny. Biol. Rev. Camb. Philos. Soc., 41(4):587-640, 1966.

Hammer, Ø.; Harper, D. A. T. \& Ryan, P. D. PAST v. 2.17c. Palaeontol. Electron., 4(1), 1-229, 2001

Hu, Y.; Hu, S.; Wang, W.; Wu, X.; Marshall, F. B.; Chen, X.; Hou, L. \& Wang, C. (2014). Earliest evidence for commensal processes of cat domestication. Proc. Natl. Acad. Sci. U. S. A., 111(1):116-20, 2014.

Integrated Taxonomic Information System Online Database. Felis silvestris Schreber, 1777. Integrated Taxonomic Information System Online Database, ITIS Report, 2020. Disponible en: https://www.itis.gov/servlet/ SingleRpt/SingleRpt?search_topic $=$ TSN $\&$ search_value $=180589 \#$ \#ull

Klingenberg, C. P. MorphoJ: an integrated software package for geometric morphometrics. Mol. Ecol. Resour., 11(2):353-7, 2011.

Klingenberg, C. P. Studying morphological integration and modularity at multiple levels: concepts and analysis. Philos. Trans. R. Soc. Lond. B Biol. Sci., 369(1649):20130249, 2014.

Künzel, W.; Breit, S. \& Oppel, M. Morphometric investigations of breed-specific features in feline skulls and considerations on their functional implications. Anat. Histol. Embryol., 32(4):218-23, 2003.
Menotti-Raymond, M.; David, V. A.; Pflueger, S. M.; Lindblad-Toh, K.; Wade, C. M.; O'Brien, S. J. \& Johnson, W. E. Patterns of molecular genetic variation among cat breeds. Genomics, 91(1):1-11, 2008.

Ottoni, C.; Van Neer, W.; De Cupere, B.; Daligault, J.; Guimaraes, S.; Peters, J.; Spassov, N.; Prendergast, M. E.; Boivin, N.; Morales-Muñiz, A.; et al. The palaeogenetics of cat dispersal in the ancient world. Nat. Ecol. Evol., 1:0139, 2017.

Parsons, K. J.; Rigg, A.; Conith, A. J.; Kitchener, A. C.; Harris, S. \& Zhu, H. Skull morphology diverges between urban and rural populations of red foxes mirroring patterns of domestication and macroevolution. Proc. Biol. Sci., 287(1928):20200763, 2020.

Pitakarnnop, T.; Buddhachat, K.; Euppayo, T.; Kriangwanich, W. \& Nganvongpanit, K. Feline (Felis catus) skull and pelvic morphology and morphometry: gender-related difference? Anat. Histol. Embryol., 46(3):294303, 2017.

Rohlf, F. J. The tps series of software. Hystrix Ital. J. Mamm., 26(1):9-12, 2015.

Sánchez-Villagra, M. R.; Segura, V.; Geiger, M.; Heck, L.; Veitschegger, K. \& Flores, D. On the lack of a universal pattern associated with mammalian domestication: differences in skull growth trajectories across phylogeny. R. Soc. Open Sci., 4(10):1-12, 2017.

Sañudo, C. Valoración Morfológica de los Animales Domésticos. Madrid, Sociedad Española de Zootecnólogos, Ministerio de Medio Ambiente y Medio Rural y Marino, Gobierno de España, 2009.

Sebastiani, A. M. \& Fishbeck, D. W. Mammalian Anatomy - The Cat. Englewood, Morton Publishing Co., 2005.

Serpell, J. A. Domestication and History of the Cat. In: Turner, D. C. \& Bateson, P. (Eds.). The Domestic Cat: The Biology of its Behaviour. $3^{\text {rd }}$ ed. Cambridge, Cambridge University Press, 2014. pp.83-100.

Stacharski, M.; Pe zin’ska, K.; Wróblewska, M.; Wojtas, J. \& Baranowski, P. The biometric characteristics of domestic cat skull in three stages of its growth: juvenile, subadult and adult. Acta Sci. Pol., Zootech., 9(3):65-78, 2015.

Stefen, C. \& Heidecke, D. Craniometric variability of European wildcats (Felis silvestris Schreber, 1777) in the Harz Mountains and comparison to other populations. Hercynia, 44(2):253-85, 2011.

Stefen, C. \& Heidecke, D. Ontogenetic changes in the skull of the European wildcat (Felis silvestris Schreber, 1777). Vertebr. Zool., 62(2):281-94, 2012.

Vigne, J. D.; Evin, A.; Cucchi, T.; Dai, L.; Yu, C.; Hu, S.; Soulages, N.; Wang, W.; Sun, Z.; Gao, J.; et al. Earliest "domestic" cats in China identified as leopard cat (Prionailurus bengalensis). PLoS One, 11(1):e0147295, 2016.

von Cramon-Taubadel, N.; Frazier, B. C. \& Mirazón Lahr, M. The problem of assessing landmark error in geometric morphometrics: theory, methods, and modifications. Am. J. Phys. Anthropol., 134(1):24-35, 2007.

Webster, M. \& Sheets, H. D. A Practical Introduction to Landmark-Based Geometric Morphometrics. In: Alory, J. \& Hunt, G. (Eds.), Quantitative Methods in Paleobiology. Boulder, The Paleontological Society, 2010.

Zelditch, M. L.; Swiderski, D. L.; Sheets, D. H. \& Fink, W. Geometric Morphometrics for Biologists: A Primer. Burlington, Elsevier, 2004.

Dirección para correspondencia:

Pere M. Parés-Casanova

Department de Ciència Animal, ETSEA

Universitat de Lleida

Av. Rovira Roure, 191, 25198

Lleida

Cataluña - ESPAÑA

E-mail: peremiquelp@ca.udl.cat 